\title{
An fMRI study of multimodal selective attention in schizophrenia
}

Andrew R. Mayer, Faith M. Hanlon, Terri M. Teshiba, Stefan D. Klimaj, Josef M. Ling,

Andrew B. Dodd, Vince D. Calhoun, Juan R. Bustillo and Trent Toulouse

\section{Background}

Studies have produced conflicting evidence regarding whether cognitive control deficits in patients with schizophrenia result from dysfunction within the cognitive control network (CCN; top-down) and/or unisensory cortex (bottom-up).

\begin{abstract}
Aims
To investigate $\mathrm{CCN}$ and sensory cortex involvement during multisensory cognitive control in patients with schizophrenia.
\end{abstract}

\section{Method}

Patients with schizophrenia and healthy controls underwent functional magnetic resonance imaging while performing a multisensory Stroop task involving auditory and visual distracters

\section{Results}

Patients with schizophrenia exhibited an overall pattern of response slowing, and these behavioural deficits were associated with a pattern of patient hyperactivation within auditory, sensorimotor and posterior parietal cortex. In contrast, there were no group differences in functional activation within prefrontal nodes of the $\mathrm{CCN}$, with small effect sizes observed (incongruent-congruent trials). Patients with schizophrenia also failed to upregulate auditory cortex with concomitant increased attentional demands.

\section{Conclusions}

Results suggest a prominent role for dysfunction within auditory, sensorimotor and parietal areas relative to prefrontal CCN nodes during multisensory cognitive control.

\section{Declaration of interest}

J.R.B. reported receiving an honorarium for consulting for Otsuka Pharmaceuticals.

\section{Copyright and usage}

(C) The Royal College of Psychiatrists 2015.
Humans are constantly bombarded with streams of information from multiple sensory modalities (e.g. auditory and visual) that must be rapidly processed to execute appropriate behaviours. ${ }^{1}$ Cognitive control refers to the ability to facilitate goal-directed behaviours while suppressing inappropriate and/or distracting stimuli and behaviours, and has recently been expanded to include working memory paradigms. $^{2}$ Although deficits in cognitive control are commonly reported in patients with schizophrenia, ${ }^{3}$ the majority of previous schizophrenia studies have utilised unisensory stimuli, ${ }^{4,5}$ with only a single study employing a more realistic multisensory approach. ${ }^{6}$ Unisensory studies may not adequately capture the complex environment that is typical in occupational and interpersonal settings where patients with schizophrenia have been shown to exhibit deficits associated with cognition. Thus, to understand the underlying neuronal processes of cognitive control in real-world environments, a multisensory approach is needed.

There are two potential theories regarding cognitive control deficits in patients with schizophrenia. The first theory suggests that poor performance in patients results from dysfunction within the cognitive control network $(\mathrm{CCN})$ during trials with conflicting (i.e. incongruent trials) relative to non-conflicting (i.e. congruent trials) information. ${ }^{6-8}$ The core $\mathrm{CCN}$ in healthy controls includes the dorsal medial prefrontal cortex, dorsolateral and ventrolateral prefrontal cortex (lateral prefrontal cortex), anterior insula and the inferior parietal lobes. ${ }^{9}$ Previous neuroimaging studies have reported abnormalities within the lateral prefrontal cortex, dorsal medial prefrontal cortex, posterior parietal cortex, lateral temporal cortex and thalamus for patients with schizophrenia relative to healthy controls across a variety of tasks. ${ }^{10}$ However, reduced ${ }^{7,11}$ and increased ${ }^{12,13}$ prefrontal activation have been reported, as well as a combination of both findings. ${ }^{14,15}$ The single imaging study examining multisensory cognitive control in patients with schizophrenia reported hypoactivation within lateral prefrontal cortex, temporoparietal juncture and medial temporal regions. ${ }^{6}$

Although top-down allocation of attention necessitates the recruitment of the $\mathrm{CCN}$, competent performance is also dependent on the processing of stimuli within the sensory cortex. A second theory therefore suggests that dysfunction within sensory cortex contributes to downstream cognitive control deficits in patients with schizophrenia. ${ }^{4,5}$ These deficits have been observed in both auditory and visual steady state responses, ${ }^{16}$ mismatch negativity, ${ }^{17}$ abnormal auditory gating ${ }^{18}$ and reduced visual evoked potentials. ${ }^{5}$ Conversely, hyperactivation of auditory $\operatorname{cortex}^{19}$ and primary visual cortex ${ }^{20}$ has also been reported, suggestive of over-processing of sensory stimuli. Finally, a direct interaction exists between attentionally demanding multisensory conditions (requiring more cognitive control) and the degree of neuronal activation within unisensory cortex. These attentionrelated modulations (ARMs) include enhanced neural responses (i.e. upregulation) within primary and secondary sensory cortices for the attended stimuli and suppressed responses for the ignored stimuli, the appearance of new waveforms, as well as more synchronous neuronal spiking. ${ }^{1,21,22}$

Thus, multisensory cognitive control tasks are uniquely poised to distinguish between deficits resulting from top-down abnormalities within the CCN (incongruent auditory/visual trials $v$. congruent auditory/auditory or visual/visual trials), basic deficits in neurovascular properties within unisensory cortex, and the direct modulation of unisensory cortex (ARMs). We hypothesised that patients with schizophrenia would exhibit deficits within the CCN specifically during cognitive control (incongruent $v$. congruent trials), whereas deficits within the unisensory cortex would be seen generally (across both incongruent and congruent trials). In addition, we predicted that patients with schizophrenia would fail to exhibit upregulation of unimodal 
sensory cortical areas (ARMs) to attended stimuli at increasing cognitive loads (high frequency stimuli relative to low frequency stimuli) as has been observed in healthy populations. ${ }^{1}$

\section{Method}

\section{Participants}

Thirty-seven clinically stable patients with schizophrenia and 37 age- and gender-matched healthy controls were included. Data from one patient were lost secondary to acquisition problems, and one patient was an outlier (three standard deviations) relative to their cohort on two of six motion parameters (frame-wise displacement). Two patients with schizophrenia performed below chance levels (based on a binomial distribution) on the task, leaving a total of 33 patients with schizophrenia (29 males; 36.0 years old $($ s.d. $=13.6)$ ) and 33 matched healthy controls $(29$ males; 34.6 years old $($ s.d. $=12.6))$ for final analyses. Informed consent was provided according to institutional guidelines.

Inclusion criteria for patients with schizophrenia included a diagnosis of schizophrenia based on the Structured Clinical Interview for DSM-IV-TR and age of 18-65 years. Most patients with schizophrenia $(32 / 33)$ were on a variety of antipsychotic medications (28/32 patients atypical) such that olanzapine equivalency scores were calculated. ${ }^{23}$ Exclusion criteria for both groups included a history of neurological disorder, head trauma with loss of consciousness greater than $5 \mathrm{~min}$, mental retardation, inadequate hearing (tested with the calibrated finger rub auditory screening test), active substance dependence or misuse within the past year and lifetime history of dependence or use within the last 12 months of phencyclidine, amphetamines or cocaine. Additional exclusion criteria for healthy controls included a current or past psychiatric disorder (with the exception of one lifetime depressive episode), depression or antidepressant use within the past 6 months, lifetime antidepressant use of more than 1 year, and history of a psychotic disorder in a first-degree relative. All participants refrained from smoking for at least $1 \mathrm{~h}$ before scanning.

\section{Neuropsychological and clinical assessment}

All participants completed the Wechsler Test of Adult Reading (WTAR). Patients with schizophrenia completed the Measurement and Treatment Research to Improve Cognition in Schizophrenia (MATRICS) battery, Positive and Negative Syndrome Scale (PANSS), Calgary Depression Scale, Clinical Global Impression, Fagerstrom Test for Nicotine Dependence (FTND), a modified version of the Simpson Angus Scale for Parkinsonism (SAS), Abnormal Involuntary Movements Scale (AIMS) for tardive dyskinesia, Barnes Akathisia Scale (BAS), urine drug screening and the UCSD Performance Based Skills Assessment test (UPSA-2). Please see online supplemental Methods for references associated with all clinical assessments.

\section{Task}

The task was identical to previous publications. ${ }^{24}$ Congruent or incongruent multisensory (auditory and visual) numeric stimuli (Fig. 1A and 1B) were simultaneously presented at either low $(0.33 \mathrm{~Hz} ; 3$ trials/block) or high $(0.66 \mathrm{~Hz} ; 6$ trials/block) frequency rates in 10-second blocks. For each block, the stream of target numbers (one, two or three) was preceded by a cue word, 'HEAR' or 'LOOK'. If the cue was 'HEAR', participants were instructed to respond via a right-handed button press to aurally presented target stimuli and ignore simultaneously presented visual numbers (attend-auditory condition). When the cue was 'LOOK', visually presented stimuli were the targets and participants were instructed to ignore auditory stimuli (attend-visual condition). The inter-block intervals varied between 8,10 and $12 \mathrm{~s}$ to decrease temporal expectations and permit modelling of the baseline response. Order of blocks was pseudorandom, with a total of 432 trials presented across six separate imaging runs. Median reaction times were used as measures of central tendency to reduce the influence of skew. Accuracy was analysed using ranking of percentage of errors in each trial type to account for deviations in normality. $2 \times 2 \times 2$ (group (patients with schizophrenia $v$. healthy controls) $\times$ condition (congruent $v$. incongruent $) \times$ frequency $(0.33 \mathrm{~Hz} v .0 .66 \mathrm{~Hz})$ ) mixed-measures analysis of variance (ANOVAs) were conducted separately on attend-auditory and attend-visual conditions for both accuracy and response time data.

\section{Magnetic resonance imaging and statistical analyses}

Magnetic resonance imaging (MRI) data including $\mathrm{T}_{1}$ images and echo-planar imaging (EPI) were collected on a Siemens 3T Trio Tim scanner with a 12 channel head coil. EPI data were collected using a single-shot, gradient-echo sequence (repetition time $(\mathrm{TR})=2000 \mathrm{~ms}$; voxel size: $3.75 \times 3.75 \times 4.55 \mathrm{~mm}$; see online supplemental Methods). Functional imaging maps were calculated using the Analysis of Functional NeuroImages (AFNI) software (http://afni.nimh.nih.gov). Standard pre-processing steps included motion correction, slice timing correction, smoothing $(8 \mathrm{~mm}$ full-width at half-max Gaussian kernel) and spatial normalisation. A voxel-wise deconvolution analysis generated a single haemodynamic response function (HRF) for each trial-type relative to baseline (visual fixation plus baseline gradient noise) and was based on the first $22 \mathrm{~s}$ post-stimulus onset. Error trials were modelled separately. ${ }^{25}$ Percent signal change (PSC) for correct trials was calculated by summing beta coefficients for images occurring $6-14 \mathrm{~s}$ post-cue onset and dividing by the average model intercept.

Similar to accuracy and response time data, two parallel whole-brain voxel-wise, $2 \times 2 \times 2$ (group (patients with schizophrenia $v$. healthy controls) $\times$ condition (congruent $v$. incongruent $) \times$ frequency $(0.33 \mathrm{~Hz} v .0 .66 \mathrm{~Hz}))$ mixed-measures analysis of covariance (ANCOVAs) were conducted on auditory and visual modalities separately using 3dMVM in AFNI. In this analytic framework, our predictions of increased abnormalities during cognitive control are specifically tested by the group $x$ condition (increased deficits for patients with schizophrenia on incongruent trials) and group $\times$ condition $\times$ frequency (worse performance under higher cognitive loads) interactions, whereas our prediction of increased difficulty with processing multisensory stimuli is tested by the main effect of group. All voxel-wise results were corrected for false positives at $P<0.05$ based on 10000 Monte-Carlo simulations implemented in AFNI (cluster level= parametric threshold $P<0.005$; minimum cluster size $2431 \mu \mathrm{l}$ ). Clusters that survived false positive correction but exhibited greater than $75 \%$ overlap with a white matter/ventricular exclusion map were also excluded (see online supplemental Methods).

\section{ARMs analyses}

Individual $T_{1}$ data were segmented through the FreeSurfer reconstruction pipeline, with regions of interest (ROI) defined by standard labels (see online supplemental Methods). To calculate how unisensory cortex was attentionally modulated in the presence of identical sensory stimulation (ARMs), PSC data were subtracted in the expected direction of positive modulation for auditory (attend-auditory trials - attend-visual trials) and visual (attend-visual trials - attend-auditory trials) cortex for each frequency, collapsing across congruent and incongruent trials. This was followed by $2 \times 2$ (group $\times$ frequency) ANOVAs and 


\section{A) AV Task}

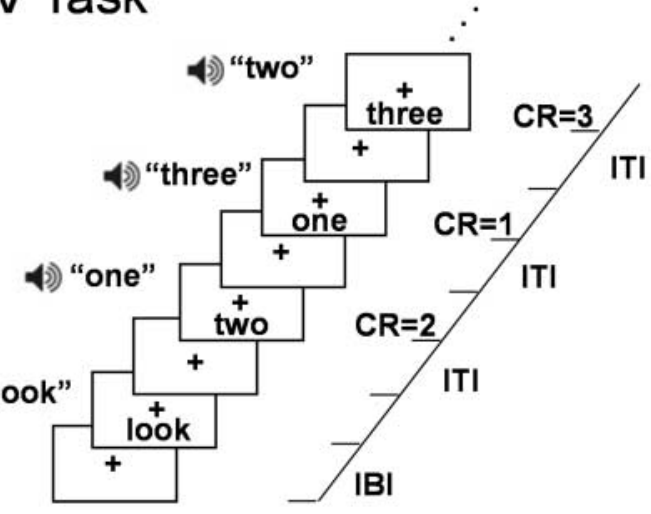

C) AV RT

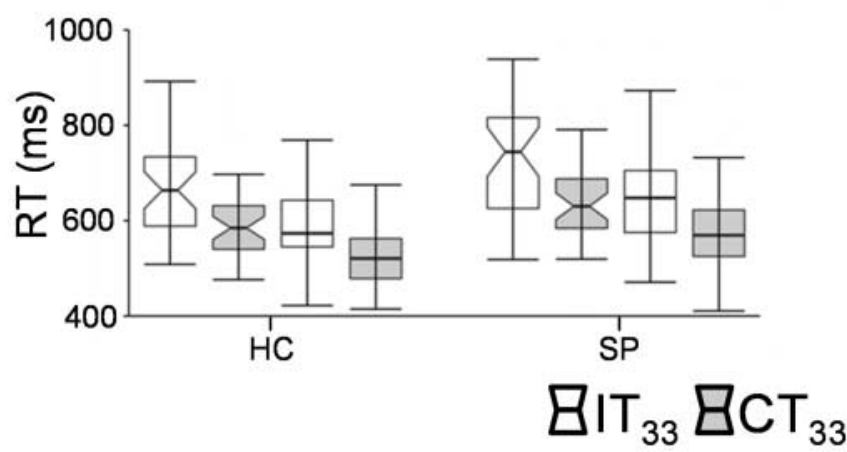

B) AA Task

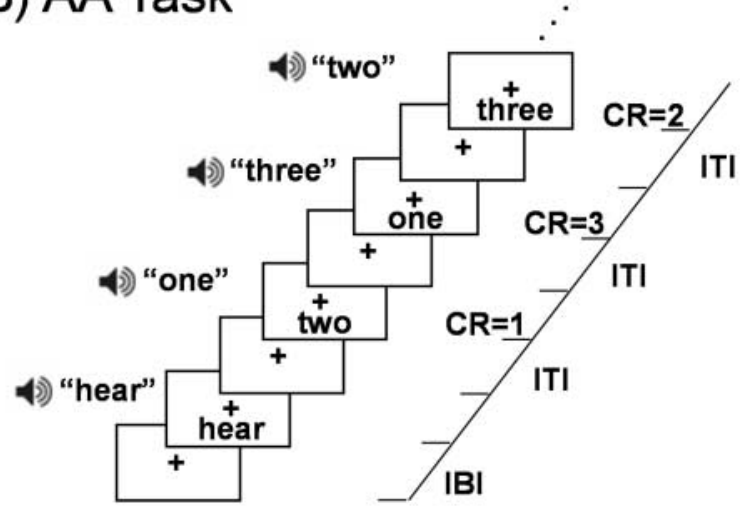

D) AA RT

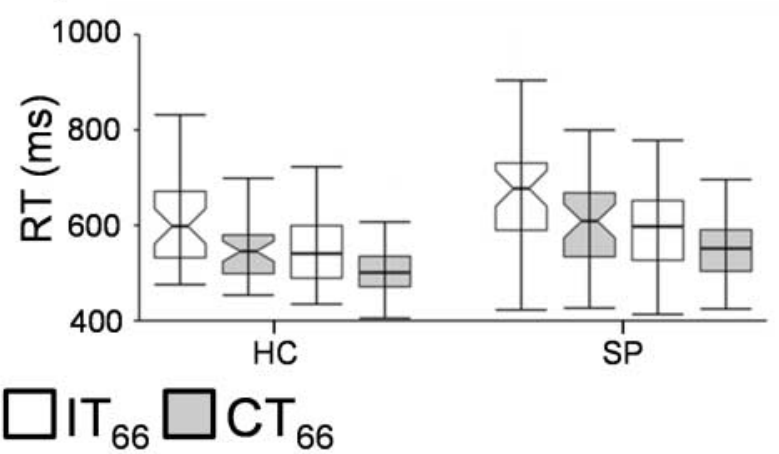

Fig. 1 The first row (Panels A and B) presents a diagrammatic representation of the attend-visual (AV) and attend-auditory (AA) tasks, which were determined by presence of a cue word ( $A V=$ 'LOOK'; $A A=$ 'HEAR') at the start of the trial. Incongruent trial types are depicted for both conditions, with the right side of each panel indicating the expected correct responses (CR) and inter-trial (ITI) and inter-block (IBI) intervals. The bottom row of the figure presents box-and-whisker plots for reaction times (RT) in AV (Panel C) and AA (Panel D).

Data are presented separately for healthy controls (HC) and patients with schizophrenia (SP), with incongruent (IT; white boxes) and congruent (CT; grey boxes) trials of the two different stimulation frequency types $(0.33 \mathrm{~Hz}$ notched boxes; $0.66 \mathrm{~Hz}$ unnotched boxes) also presented.

The centre line of each box plot represents the median RT, with whiskers representing the first value more extreme than 1.5 times

the interquartile range. As evident from the figure, main effects of congruency $(\mathrm{IT}>\mathrm{CT})$, frequency $(0.33>0.66 \mathrm{~Hz})$ and group $(\mathrm{SP}>\mathrm{HC})$ were present within the behavioural data.

one-sample $t$-tests to ensure that resulting subtraction maps were not equivalent to the null distribution (see online supplemental Methods). The group $\times$ frequency interaction specifically tested the hypothesis that patients with schizophrenia would fail to exhibit ARMs at increasing cognitive loads (faster rate of stimulus presentation). Multivariate analysis of covariance (MANCOVAs) were performed separately for primary and secondary auditory and visual cortex volumes using intracranial volume (ICV) as the covariate.

\section{Results}

\section{Demographics and clinical data}

There were no significant differences in age between the two groups $(P>0.10)$. Significant group differences were observed in education $\left(t_{64}=2.1, \quad P<0.05\right)$ and estimate of pre-morbid intelligence $\left(t_{40.5}=2.6, P<0.05\right)$, with patients with schizophrenia exhibiting lower estimated intelligence than healthy controls. See Table 1 for remainder of clinical demographics.

\section{Multisensory selective attention task behavioural data}

The ranked accuracy data were analysed separately for attendauditory and attend-visual trails using $2 \times 2 \times 2$ (group (patients with schizophrenia $v$. healthy controls) $\times$ condition (congruent $v$. incongruent $) \times$ frequency $(0.33 \mathrm{~Hz} v .0 .66 \mathrm{~Hz}))$ ANOVAs. There were no significant effects for any factors or interactions in the attend-visual trials. The three-way interaction was significant for the attend-auditory condition $\left(F_{1,64}=7.9, P<0.05\right)$, with follow-up analyses indicating that patients were less accurate on high-frequency, incongruent trials.

During attend-visual trials (Fig. 1C), ANOVA results for reaction time data indicated significant main effects of condition $\left(F_{1,64}=87.7, P<0.05\right)$, frequency $\left(F_{1,64}=126.7, P<0.05\right)$ and group $\left(F_{1,64}=6.1, P<0.05\right)$, with faster response times for congruent $(548.0 \mathrm{~ms} \quad($ s.d. $=78.4))$ relative to incongruent $(607.8 \mathrm{~ms}(\mathrm{s.d} .=106.0))$ and high $(546.8 \mathrm{~ms}($ s.d. $=85.2))$ relative to low $(609.0 \mathrm{~ms}($ s.d. $=98.7))$ frequency trials. Healthy controls $(551.7 \mathrm{~ms}$ (s.d. $=73.2)$ ) also responded faster to targets relative to patients with schizophrenia $(604.1 \mathrm{~ms}($ s.d.=97.4)). Nonsignificant trends were observed for condition $\times$ group $\left(F_{1,64}=2.9, \quad P=0.096\right)$ and frequency $\times$ condition $\left(F_{1,64}=3.1\right.$, $P=0.082)$ interactions. The non-significant condition $\times$ group interaction indicated increased slowing for patients with schizophrenia while ignoring auditory stimuli (incongruentcongruent trials $70.6 \mathrm{~ms}$ (s.d. $=57.5)$ ) relative to healthy controls (incongruent-congruent trials $49.0 \mathrm{~ms}(\mathrm{~s} . \mathrm{d} .=45.6)$ ) beyond general response slowing.

For attend-auditory trials (Fig. 1D), significant main effects of both condition $\left(F_{1,64}=78.1, \quad P<0.05\right)$ and frequency $\left(F_{1,64}=107.4, P<0.05\right)$ were noted, with participants responding 
more rapidly to congruent $(586.6 \mathrm{~ms}($ s.d. $=86.5))$ compared with incongruent $(657.6 \mathrm{~ms}$ (s.d. $=104.9))$ trials, as well as to higher $(582.7 \mathrm{~ms}($ s.d. $=87.9))$ relative to lower $(661.5 \mathrm{~ms}($ s.d. $=102.7))$ frequency trials. In addition, the main effect of group was also significant $\left(F_{1,64}=6.6, P<0.05\right)$, with patients with schizophrenia $(649.5 \mathrm{~ms}(\mathrm{~s} . \mathrm{d} .=98.3))$ responding more slowly to attend-auditory trials relative to healthy controls $(594.7 \mathrm{~ms}($ s.d. $=73.8))$. No interaction effects were significant $(P<0.10)$.

\section{Motion parameter analyses}

Two multivariate analyses of variance (MANOVAs) were performed to examine potential group differences in frame-wise displacement for all six motion parameters. Although the group effect was not significant for the translational motion MANOVA $(P>0.10)$, there was a significant effect for the rotational motion MANOVA $\left(F_{3,62}=4.04, P<0.05\right)$, with univariate measures indicating significantly increased motion for patients with schizophrenia (pitch: $F_{1,64}=8.29, P<0.05 ;$ yaw: $F_{1,64}=7.67$, $P<0.05)$. The utilisation of covariates in the presence of group differences is actively debated, ${ }^{26}$ but motion can produce spurious activation. ${ }^{27}$ Primary functional analyses were therefore performed with frame-wise displacement as a covariate, whereas analyses without frame-wise displacement are reported in the online supplement. In addition, more aggressive strategies ${ }^{27}$ for eliminating individuals with excessive motion were also evaluated (see online supplemental Results).

\section{Attend-visual fMRI results}

A voxel-wise, $2 \times 2 \times 2$ (group $\times$ condition $\times$ frequency) mixedmeasures ANCOVA was performed for the attend-visual condition. Contrary to $a$ priori predictions, neither the group $\times$ condition nor the group $\times$ condition $\times$ frequency interaction was significant. Regions exhibiting increased activation for incongruent relative to congruent trials (CCN; see online supplement Fig. DS1 for effects collapsed across group and Fig. DS2 for individual group effects) included bilateral dorsal medial prefrontal cortex (Brodmann areas (BAs) 6/9/24/32/33), bilateral anterior insula, lateral prefrontal cortex extending into the precentral gyrus (left BAs $4 / 6 / 8 / 9 / 10 /$ 13/44/45/46/47; right BAs 6/9/13/44/45/46/47), bilateral middle and posterior superior temporal gyri/sulci extending into inferior parietal lobule (BAs 13/21/22/39/40), the left posterior parietal cortex (BAs 19/39/40) and left precuneus/posterior cingulate gyrus (BAs 7/31). Increased activation for incongruent trials was also observed within the bilateral thalamus, basal ganglia and midbrain nuclei during the attend-visual condition.

A main effect of group was also present in several cortical regions during attend-visual conditions (Fig. 2). The findings were represented by two primary patterns of patient hyperactivation and potentially failed deactivation, supporting the hypothesis of a generalised deficit in unisensory cortex activation in patients with schizophrenia. Specifically, patient hyperactivation was observed in the right (BAs 13/38/40/43) auditory cortex, as well as left (BAs 2/5/7/40) and right (BAs /2/3/4/5/6/7/40) sensorimotor cortex, posterior parietal cortex and precuneus. For the second pattern, healthy controls exhibited deactivation within right extrastriate primary visual cortex (BAs 18/19/29/30/31), whereas patients with schizophrenia exhibited baseline activity. Finally, healthy controls displayed increased activation in lobules VII and VIII of the left cerebellum relative to patients with schizophrenia. Similar results were obtained when individuals with greater than 0.50 mean frame-wise displacement were excluded from analyses. Additional regions of patient hyperactivation (left auditory cortex) and healthy control deactivation (left visual cortex, paracentral lobule and cingulate cortex) were observed when frame-wise displacement was excluded from the model as a covariate (see online supplemental Results; Fig. DS3). Qualitative examination of the HRF indicated both an increased amplitude and duration of response for patients with schizophrenia, with little evidence of a post-undershoot (online Fig. DS4).

Due to null effects and concerns about insufficient power, supplemental analyses were also conducted on the CCN to determine effect sizes. Specifically, regions within the CCN were first identified by comparing the intersection of the within-participant comparisons for the contrast of incongruent $v$. congruent trials (see online Fig. DS2 for individual group maps). Table 2 indicates

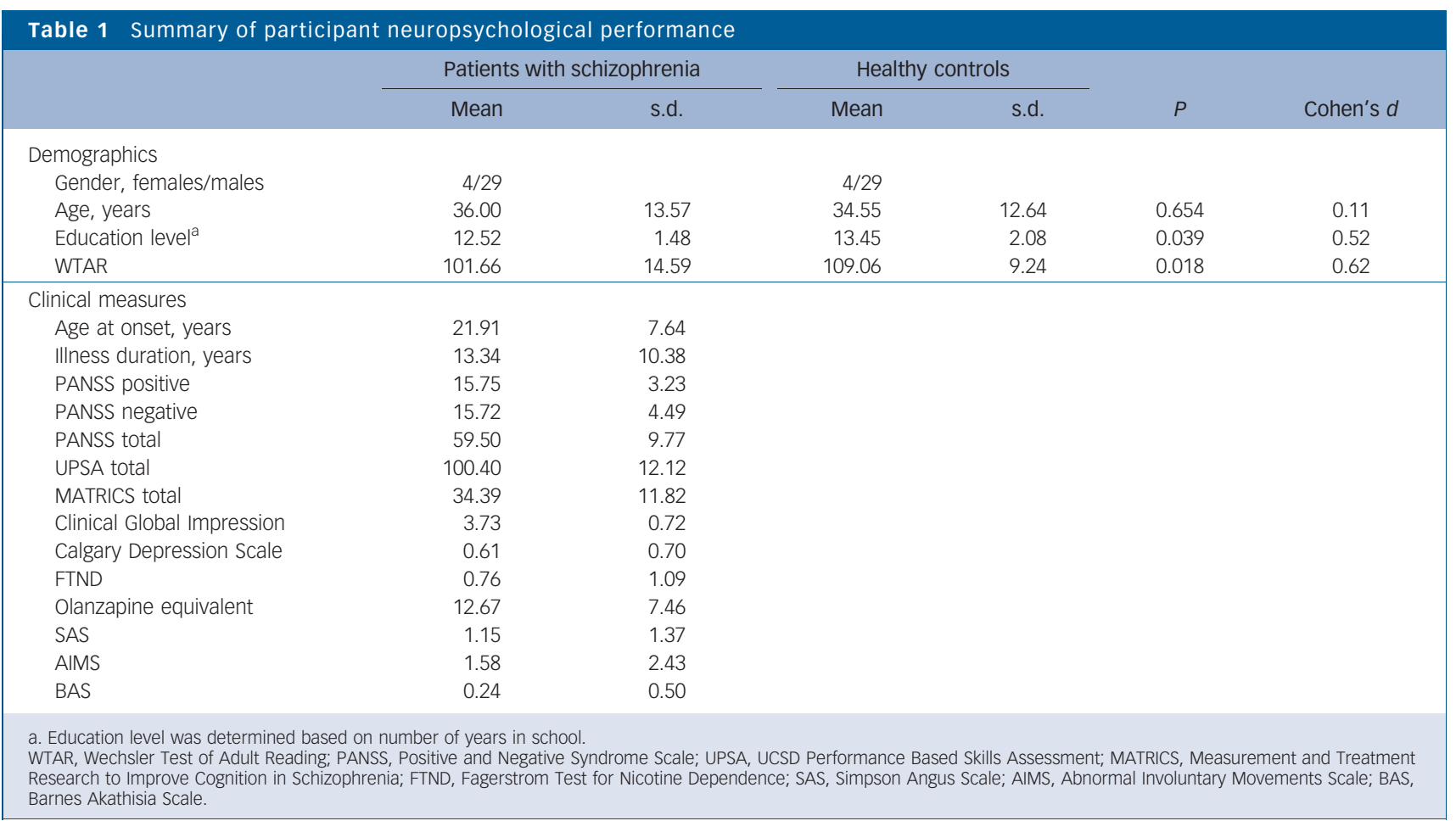




\section{A) AV Group Effect}
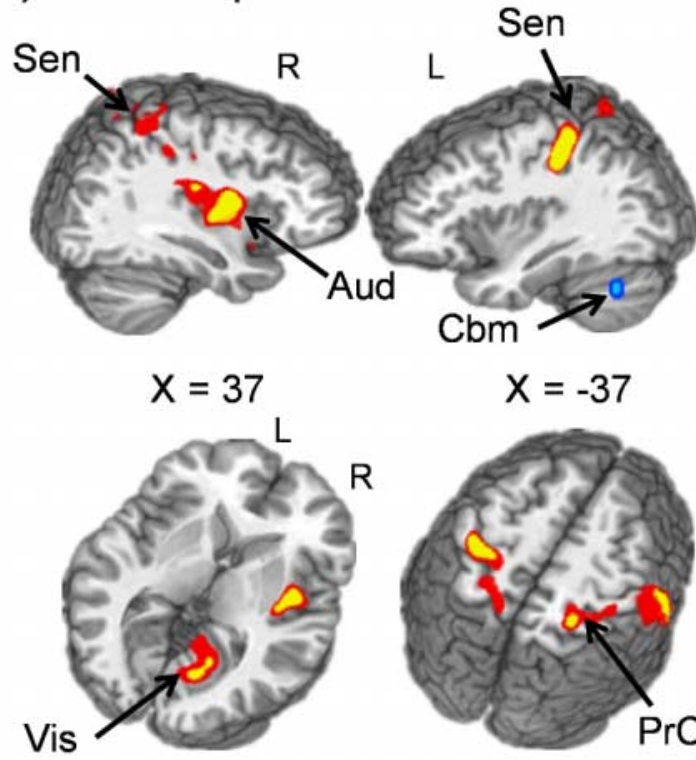

$Z=4$

SP

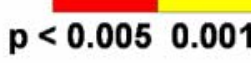

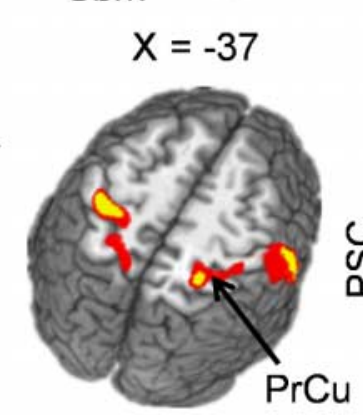

$Z=52$
HC

B) Group ROI

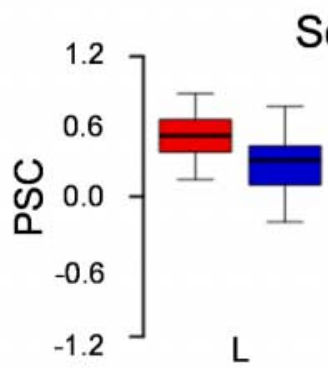

Sen
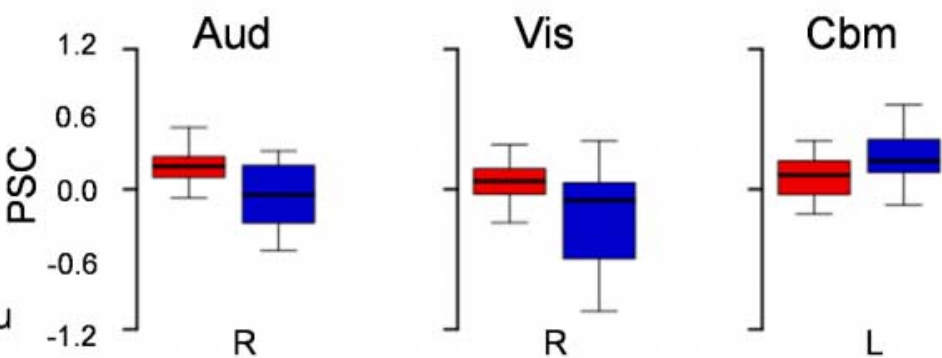

SP $\square \mathrm{HC}$

Fig. 2 Panel A displays the regions of the brain showing significant group differences between patients with schizophrenia (SP - warm colours) and healthy controls ( $\mathrm{HC}$ - cool colours) during the attend-visual (AV) condition when using mean frame-wise displacement as a covariate. Locations of the sagittal $(\mathrm{X})$ and axial $(\mathrm{Z})$ slices are given according to the Talairach atlas for the left (L) and right (R) hemispheres. Panel B displays box-and-whisker plots of the mean percent signal change (PSC) for selected regions of interest. Patients with schizophrenia showed increased activation relative to healthy controls within the right auditory cortex (Aud), bilateral sensorimotor cortex (Sen), posterior parietal cortex and precuneus (PrCu). Healthy controls also exhibited deactivation within right extrastriate primary visual cortex (Vis), whereas patients with schizophrenia exhibited baseline activity. Finally, healthy controls displayed increased activation in lobules VII and VIII of the left cerebellum (Cbm) relative to patients with schizophrenia.

the regions of common activation across both patients with schizophrenia and healthy controls for this contrast as well as the respective effects sizes. For all regions within the $\mathrm{CCN}$, effect sizes were typically in the small range (range -0.22 to 0.03 ).

The group $\times$ frequency interaction (see online Fig. DS5) was significant within the culmen and declive bilaterally and the right posterior parietal cortex (BAs 7/40). Simple effects testing indicated a consistent pattern in which patients with schizophrenia exhibited increased activation within both of these regions as a function of stimulus frequency (both $P s<0.05$ ), whereas healthy controls' levels remained at the same level of activation regardless of stimulation frequency (both $P_{s}>0.10$ ).

The main effect of frequency and other second-order interactions (attend-visual and attend-auditory conditions) are not central to the current paper and are presented in the online supplementary Results.

\section{Attend-auditory fMRI results}

Similar to the attend-visual results, neither the group $\times$ condition nor the group $\times$ condition $\times$ frequency interaction was significant for attend-auditory trials. There were two different networks that exhibited either increased activation during incongruent trials (CCN) or increased activation during congruent trials (sensory cortices) for attend-auditory trials (see online Fig. DS6 for effects collapsed across group and online Fig. DS7 for individual group effects). Increased activation for incongruent trials was observed in the bilateral anterior insula extending into the ventrolateral prefrontal cortex (BAs 13/45/47), bilateral dorsal medial prefrontal cortex (BAs 24/32) and the left dorsolateral prefrontal cortex/ precentral gyrus (BAs 4/6/9). Increased activation for incongruent trials was also observed in the posterior aspects of the left middle and posterior superior temporal gyrus/sulcus (BAs 21/22/39/40), bilateral basal ganglia, midbrain nuclei and thalamus. In contrast, regions of increased activation during congruent trials included both the bilateral 'what' (fusiform, parahippocampal gyri and lingual gyri; BAs 18/19/36/37) and 'where' (middle occipital gyri, precuneus and cuneus; BAs 7/18/19/31) visual streams, as well as bilateral secondary auditory cortex extending into the precentral gyrus (BAs 13/43/4/6) and left putamen.

For the main effect of group (Fig. 3), patients exhibited hyperactivation within right secondary auditory cortex (BAs 13/43) and bilateral sensorimotor cortex/posterior parietal cortex (inferior and superior aspects; left BAs 2/5/7/40 and right BAs 1/2/3/4/5/ $6 / 7 / 40$ ) during attend-auditory trials. In contrast, in healthy controls, hyperactivation was observed in the left cerebellum. Results remained unchanged when participants with greater than 0.50 mean frame-wise displacement were excluded from analyses. Similar to the attend-visual condition, additional clusters of patient hyperactivation (left auditory cortex) and healthy control deactivation (paracentral lobule and cingulate gyrus) were observed when frame-wise displacement was eliminated from the model. In addition, there were no differences between groups in the left cerebellum when the covariate was eliminated (online Fig. DS8). Examination of the entire HRF (online Fig. DS9) indicated a similar pattern of abnormalities as in the attend-visual condition (increased response amplitude/duration and no post-undershoot for patients with schizophrenia). 


\begin{tabular}{|lccc|}
\hline Table 2 & Effect sizes for regions of common activation within the CCN & Patients with schizophrenia & Healthy controls \\
& Size, $\mu \mathrm{L}$ & $n$ (mean) s.d. & $33(0.121) 0.134$ \\
\hline L IPFC & 19383 & $33(0.094) 0.105$ & $33(0.099) 0.120$ \\
\hline L pSTS & 7121 & $33(0.090) 0.100$ & $33(0.078) 0.134$ \\
\hline R IPFC & 2255 & $33(0.083) 0.135$ & $33(0.062) 0.109$ \\
\hline B ACC & 1853 & $33(0.056) 0.099$ & $33(0.113) 0.153$ \\
\hline L dACC/p-SMA & 1777 & $33(0.082) 0.126$ & -0.09 \\
\hline B, L, R, bilateral, left, right; IPFC, lateral prefrontal cortex; pSTS, posterior superior temporal sulcus; dACC, dorsal anterior cingulate gyrus; p-SMA, pre-supplementary motor area.
\end{tabular}

\section{A) AA Group Effect}

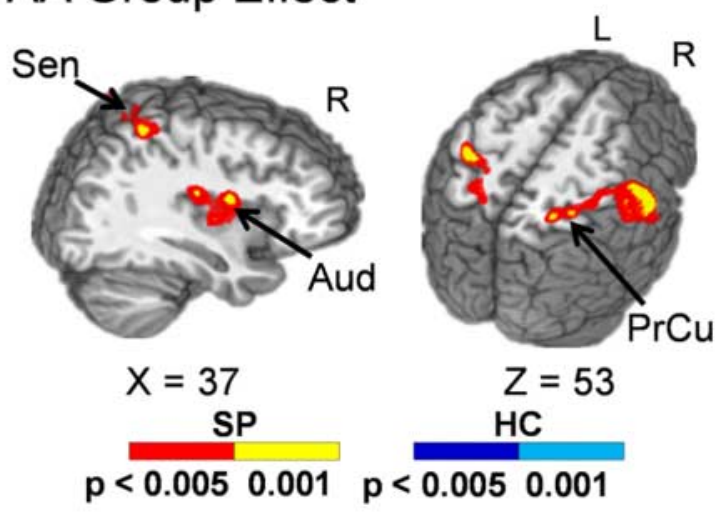

B) Group ROI

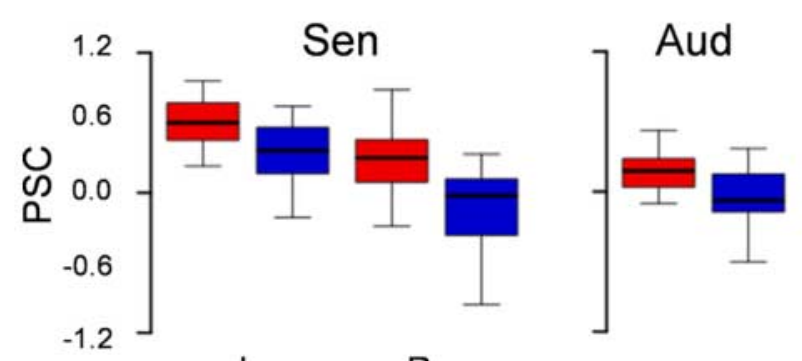

$\mathrm{L}$

$\mathrm{R}$

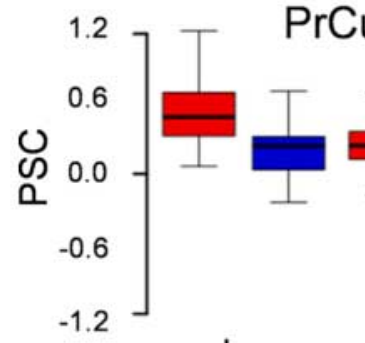

$\mathrm{L}$
(

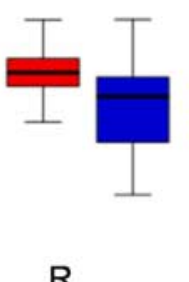

SP $\square$ HC

Fig. 3 Panel A displays the regions of the brain showing significant group differences between patients with schizophrenia (SP- warm colours) and healthy controls ( $\mathrm{HC}$ - cool colours) during the attend-auditory (AA) condition when using mean frame-wise displacement as a covariate. Locations of the sagittal $(X)$ and axia (Z) slices are given according to the Talairach atlas for the left (L) and right (R) hemispheres. Panel B presents the box-and-whisker plots for the mean percent signal change (PSC) for selected regions of interest (ROI). Increased activation for patients with schizophrenia relative to healthy controls was observed within the right secondary auditory cortex (Aud), bilateral sensorimotor cortex (Sen), posterior parietal cortex and precuneus (PrCu). In contrast, healthy control hyperactivation was observed in the left cerebellum (Cbm).

\section{ARMs analyses}

Results from two MANCOVAs indicated no significant differences in auditory or visual cortical volume between the two groups (both $P_{\mathrm{s}}>0.10$ ).

ARMs analyses $(2$ (group) $\times 2$ (frequency) ANOVAs) indicated trend-level interaction effects within the primary $\left(F_{1,64}=3.88\right.$, $P=0.053)$ and significant interaction effects within the secondary $\left(F_{1,64}=4.05, P<0.05\right)$ visual cortex (Fig. $4 \mathrm{~A}$ and $\left.4 \mathrm{~B}\right)$. Simple effects tests indicated a trend for increased ARMs for patients with schizophrenia relative to healthy controls within primary visual cortex $\left(t_{64}=-1.73, P=0.089\right)$, with significantly increased ARMs in secondary visual cortex $\left(t_{64}=-2.05, P<0.05\right)$ during highfrequency trials. There were no significant group differences for low frequency trials $(P>0.10)$. However, one-sample $t$-tests indicated that robust ARMs were not present in primary or secondary visual cortex for either group $(P>0.10)$.

As predicted, the group $\times$ frequency interaction was statistically significant for primary auditory cortex $\left(F_{1,64}=4.09, P<0.05\right)$, whereas effects were not significant for secondary auditory cortex (Fig. 4C and 4D). Follow-up simple effects testing indicated no significant $(P>0.10)$ group differences in ARMs for low frequency trials $(0.33 \mathrm{~Hz})$. In contrast, patients with schizophrenia exhibited significantly decreased ARMs $\left(t_{64}=2.09, P<0.05\right)$ for auditory trials within primary auditory cortex relative to healthy controls during high-frequency trials. One-sample $t$-tests confirmed that positive modulation (i.e. PSC $>0$ ) occurred for healthy controls within primary auditory cortex $\left(t_{32}=3.15, P<0.05\right)$ but was absent for patients with schizophrenia $(P>0.10)$ during highfrequency trials. Qualitative examination of secondary auditory cortex (Fig. 4D) also suggested differences in high-frequency trials for patients with schizophrenia relative to healthy controls. One-sample $t$-tests confirmed the presence of ARMs within secondary auditory cortex for healthy controls $\left(t_{32}=3.05\right.$, $P<0.05)$ which were absent for patients with schizophrenia $(P>0.10)$. Collectively, these findings indicate that patients with schizophrenia failed to upregulate unimodal cortical areas at increasing cognitive loads.

\section{Network, behavioural and clinical interactions}

Our next series of analyses investigated whether differences in auditory, sensorimotor and posterior parietal cortex were associated with behavioural or clinical findings. Behavioural and functional results indicated a main effect of group, such that data were first averaged across all trials (reaction time and functional activity) and then across voxels (functional data) separately for attend-auditory and attend-visual conditions. Our first set of analyses indicated a positive relationship between reaction time and auditory/parietal activation for both conditions (attendvisual: $r=0.29, P=0.02$; attend-auditory: $r=0.32, P=0.008$ ) when all participants were included in analyses. These relationships were not significant when only patients with schizophrenia were examined $(P>0.10)$. 
Two linear regressions for each condition (attend-auditory and attend-visual) evaluated the relationship between the degree of hyperactivation in these regions for patients only with (a) clinical and neuropsychological measures and with (b) measures of motor pathology. The seven independent variables in the first series of analyses included (a) MATRICS Overall Composite score, (b) UPSA total score, (c) PANSS Conceptual Disorganisation score, (d) PANSS Negative symptoms score, (e) PANSS Positive symptoms score, (f) olanzapine equivalence score and (g) FTND scores. In the second series of analyses, the three independent variables corresponded to potential motor symptoms secondary to medications (AIMS, BAS, SAS). However, the overall model was not significant in any of the four regressions (all Ps $>0.10$ ).

\section{Discussion}

The current study investigated whether unisensory cortex, CCN or ARMs would be associated with functional abnormalities during multisensory cognitive control in patients with schizophrenia. Patients had a lower educational attainment and estimate of intelligence, confirming how the typical disease course affects normal activities (e.g. school) and decreases overall cognitive functions. ${ }^{26}$ Current behavioural and functional results indicated successful parametric variation of cognitive load, with highfrequency trials resulting in the expected pattern of increased activation within bilateral unisensory (auditory, visual and sensorimotor) cortex ${ }^{24}$ and heteromodal cortex (lateral prefrontal and parietal areas), as well as increased posterior default mode network deactivation during both attend-visual and attendauditory trials. These findings are consistent with previous results $^{1,28}$ and suggest that the higher frequency trials were more attentionally demanding.

Reaction times were also faster for congruent relative to incongruent trials, and incongruent trials resulted in increased activation within the $\mathrm{CCN}^{9}{ }^{9}$ Activated nodes from the $\mathrm{CCN}$ included the dorsal medial prefrontal cortex, lateral prefrontal cortex, anterior insula, striatum, thalamus and posterior parietal cortex. In addition, the posterior superior temporal sulcus, which plays a critical role in audio-visual sensory integration, ${ }^{29}$ also showed evidence of increased activation during incongruent trials. However, the magnitude and volume of differential activation (incongruent $>$ congruent trials) appeared greater for attend-visual relative to attend-auditory condition, suggesting that ignoring auditory distracters may require increased neuronal resources. Activation was also greater for congruent relative to incongruent trials in secondary auditory cortex and primary visual cortex during attend-auditory trials, suggesting that ignoring incongruent visual stimuli did not place additional loads on sensory cortex.

The most pronounced behavioural result was an overall slowing for patients with schizophrenia relative to healthy controls during both attend-visual and attend-auditory trials, which has been previously observed for patients with schizophrenia during auditory $^{4,30}$ and visual $^{6}$ attentional tasks while attempting to ignore auditory distracters. Specific behavioural deficits in cognitive control in patients with schizophrenia were present during both the attend-auditory (i.e. increased error rate for highfrequency incongruent trials) and attend-visual trials at a trend level (i.e. increased difference in reaction time between incongruent and congruent trials). In contrast, there were no differences in functional activation within the prefrontal nodes of the CCN or thalamus. Importantly, our a priori prediction of cognitive control deficits was tested through an interaction (group $\times$ condition), which requires additional statistical power relative to a main

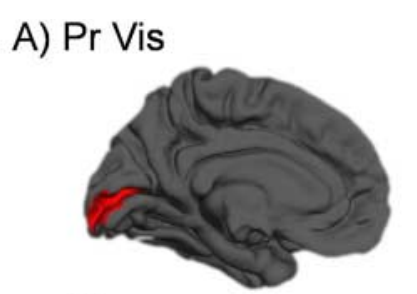

B) Sc Vis
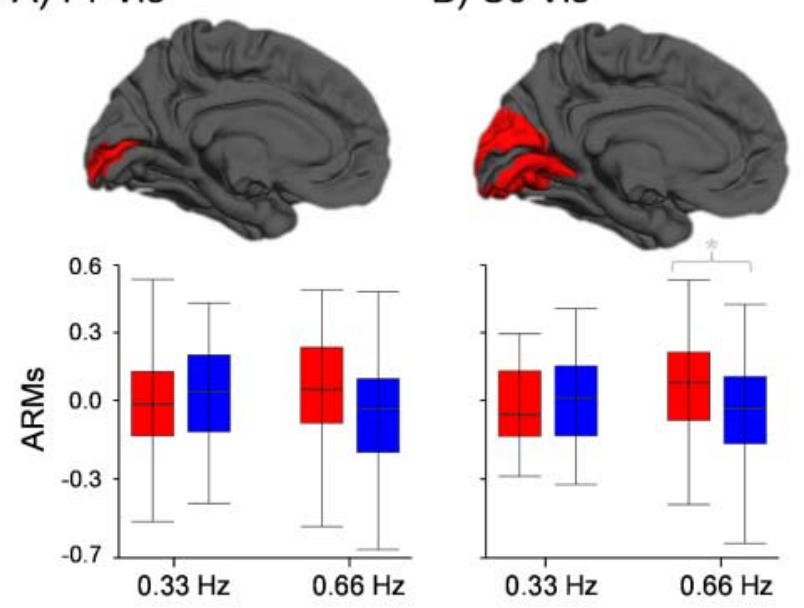

C) Pr Aud

D) Sc Aud
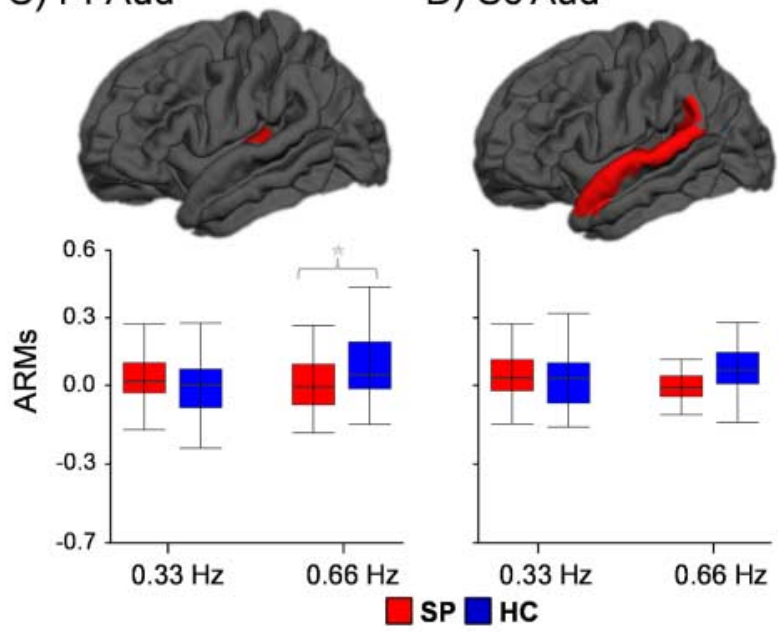

Fig. 4 This figure examines how visual (Panels A and B) and auditory (Panels $C$ and $D$ ) unisensory cortex were attentionally modulated in the presence of identical sensory stimulation (ARMs). ARM values were calculated by subtracting the percent signal change data in the expected direction of positive modulation for auditory (Aud = attend-auditory trials - attend-visual trials) and visual (Vis = attend-visual trials - attend-auditory trials) primary (Pr) and secondary (Sc) cortex, with values of zero therefore equivalent to no modulation. Box-and-whisker plots are used to demonstrate the direction of effect, with an asterisk denoting significant group differences. For the high-frequency attend-auditory trials, patients with schizophrenia (SP) failed to upregulate primary auditory cortex (Pr Aud). Although significant group differences were observed in secondary visual cortex (SC Vis) for high-frequency attend-visual trials, follow-up tests failed to establish the existence of statistically meaningful ARMs

(i.e. significantly different from the null distribution) for

either healthy controls $(\mathrm{HC})$ or patients with schizophrenia.

effect. Additional analyses indicated small effect sizes between patients with schizophrenia and healthy controls for all major nodes of the CCN, suggesting clinically unmeaningful effects rather than an under-powered sample.

There are several factors which may explain these results. First, recent studies suggest reactive cognitive control tasks such as the Stroop may not be sensitive for revealing deficits in patients with schizophrenia, ${ }^{2,14}$ and that deficits are more likely to emerge during proactive cognitive control tasks (e.g. AX continuous performance task). Similar behavioural (overall slowing with no 
group $\times$ condition interaction) and functional (similar levels of activity within the $\mathrm{CCN}$ ) results were also recently reported in a large cohort of patients with first-episode schizophrenia. ${ }^{2}$ However, others have reported deficits during reactive cognitive control tasks, ${ }^{7,11,13}$ suggesting that future studies should include both reactive and proactive cognitive control tasks to further probe this controversy. Second, the nature (multisensory $v$. unisensory) or difficulty level of the current task may have placed differential demands on cognitive resources relative to previous studies, ${ }^{10}$ potentially maximising activation within the CCN.

Consistent with a priori predictions and previous results, ${ }^{30}$ patients with schizophrenia exhibited hyperactivation in auditory, sensorimotor and posterior parietal cortex during both attendvisual and attend-auditory trials that were associated with the overall pattern of response slowing across both groups. Similar to previous results, ${ }^{19}$ qualitative examination of the HRF indicated both an increased amplitude and duration of response, with little evidence of a post-undershoot for patients with schizophrenia (online Figs. DS4 and DS9). Thus, current results indicate a larger role for dysfunction in unisensory and parietal cortex relative to the prefrontal $\mathrm{CCN}$ during multisensory reactive cognitive control. Previous findings on the direction of unisensory cortex abnormalities in patients with schizophrenia have been mixed. Several studies have reported patient hyperactivation in response to a number of different experimental conditions. ${ }^{19,31,32}$ Hyperactivation of the auditory cortex may be related to either sensory gating or habituation deficits, ${ }^{18,33}$ and hyperactivation for external stimuli has also been associated with a propensity for auditory hallucinations in healthy controls. ${ }^{34}$ Others report auditory cortex hypoactivation for external stimuli as a rationale for hallucinations, as part of the paradoxical enhancement effect. ${ }^{35}$ Hypoactivation within the lateral prefrontal cortex, fusiform gyrus, temporal-parietal juncture and hippocampus has also been reported during multisensory cognitive control. ${ }^{6}$ These contrasting findings may be secondary to the type of multisensory task and the distracters employed, as distracters in the current study were more directly relevant to the task (i.e. members of the target set).

There were no differential effects of increasing stimulus frequency within auditory or visual unisensory cortex between patients with schizophrenia and healthy controls, suggesting that basic neurovascular coupling in response to increasing sensory demands was similar across both groups. Similarly, there were no differences in primary or secondary unisensory cortical volumes between the two groups. In contrast, similar to previous studies in patients and healthy controls, ${ }^{1,35}$ current results indicated that patients with schizophrenia failed to differentially upregulate auditory cortex (ARMs analyses) under higher cognitive loads. The upregulation of unisensory cortex during multisensory tasks may facilitate the suppression of cross-modal distracters $^{21,22}$ or represent a cross-modal spread of alertness. ${ }^{36}$ Failure to 'tune' auditory cortex under different attentional demands may contribute to impaired performance in patients with schizophrenia across multiple cognitive domains ${ }^{6}$ as well as hallucinations. ${ }^{35}$ There was minimal evidence of ARMs within primary visual cortex for either healthy controls or patients with schizophrenia, although responses were increased for patients. This may be secondary to the relative lack of difficulty for ignoring visual distracters, or result from involvement of other visual pathways (outside of V1 and V2) during attentional allocation.

There are several limitations to the current study. First, patients exhibited increased head motion relative to healthy controls, and motion parameters were not used as nuisance regressors in level-one analyses per convention in mixed designs. We conservatively focused our discussions on findings that survived analyses with frame-wise displacement as a covariate, although several supplemental analyses indicated that differences in head motion did not likely affect results. Second, previous results have indicated reduced volume in unisensory cortex in patients with schizophrenia, ${ }^{37}$ and the influence of volume loss on brain activations was not examined in the current study. Third, our ability to detect relationships with clinical variables may have been restricted by the stability (low-level symptomatology) of patients and/or the chronic nature of psychosis in the current sample. Thus, current results may not generalise to other patient samples (e.g. acutely psychotic).

Fourth, healthy controls were excluded for recent depressive episodes rather than a lifetime history, and depression has been shown to affect activation within the CCN. ${ }^{38}$ Fifth, EPI produces auditory background noise secondary to gradient switching that may have differentially affected auditory cortical activity across the two groups. Finally, the full clinical and cognitive battery was not collected on healthy controls due to well-known differences between patients and controls in cognition, smoking and functional outcomes. However, none of these variables were associated with the magnitude of functional activation in the patient cohort. Controls also differed from patients in terms of educational attainment, which is typical for the disease course but may have also contributed to current results.

In summary, current results indicated overall behavioural slowing and functional abnormalities within auditory, sensorimotor/parietal areas during multisensory cognitive control. These behavioural and functional abnormalities were more pronounced while attempting to ignore auditory distracters, with patients also failing to modulate auditory cortex under different attentional demands. Thus, current results suggest that auditory dysfunction may be important for understanding multisensory cognitive control deficits in patients with schizophrenia. Future studies are needed to elucidate whether this issue extends beyond verbal stimuli and whether it can be replicated in unmedicated patients earlier in the disease course.

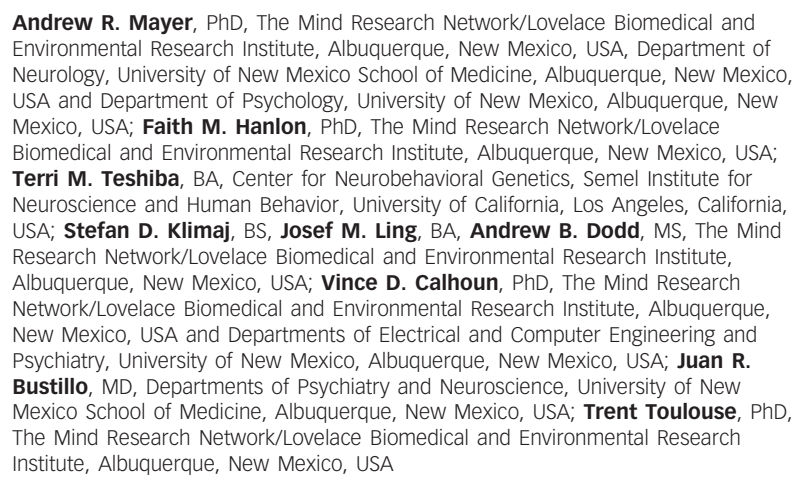

Andrew R. Mayer, PhD, The Mind Research Network/Lovelace Biomedical and Environmental Research Institute, Albuquerque, New Mexico, USA, Department of Neurology, University of New Mexico School of Medicine, Albuquerque, New Mexico, USA and Department of Psychology, University of New Mexico, Albuquerque, New Mexico, USA; Faith M. Hanlon, PhD, The Mind Research Network/Lovelace Biomedical and Environmental Research Institute, Albuquerque, New Mexico, USA; Terri M. Teshiba, BA, Center for Neurobehavioral Genetics, Semel Institute for Neuroscience and Human Behavior, University of California, Los Angeles, California, USA; Stefan D. Klimaj, BS, Josef M. Ling, BA, Andrew B. Dodd, MS, The Mind Research Network/Lovelace Biomedical and Environmental Research Institute, Albuquerque, New Mexico, USA; Vince D. Calhoun, PhD, The Mind Research Network/Lovelace Biomedical and Environmental Research Institute, Albuquerque, New Mexico, USA and Departments of Electrical and Computer Engineering and Psychiatry University of New Mexico, Albuquerque, New Mexico, USA: Juan R. Bustillo, Mexico School of Medicine, Albuquerque, New Mexico, USA; Trent Toulouse, PhD The Mind Research Network/Lovelace Biomedical and Environmental Research Institute, Albuquerque, New Mexico, USA

Correspondence: Andrew R. Mayer, PhD, The Mind Research Network, Pete \& Nancy Domenici Hall, 1101 Yale Blvd. NE, Albuquerque, NM 87106, USA; Email: amayer@mrn.org

First received 30 Jul 2014, final revision 14 Nov 2014, accepted 22 Jan 2015

\section{Funding}

This work was supported by the National Institutes of Health (grant numbers NIGMS P20GM103472(COBRE), 1R01MH101512-01A1 (R01) to A.R.M.). The funding agencies had no involvement in the study design, data collection, analyses, writing of the manuscript or decisions related to submission for publication.

\section{References}

1 Mayer AR, Franco AR, Canive J, Harrington DL. The effects of stimulus modality and frequency of stimulus presentation on cross-modal distraction. cereb Cortex 2009; 19: 993-1007. 
2 Lesh TA, Westphal AJ, Niendam TA, Yoon JH, Minzenberg MJ, Ragland JD, et al. Proactive and reactive cognitive control and dorsolateral prefrontal cortex dysfunction in first episode schizophrenia. Neuroimage Clin 2013; 2 590-9.

3 Lesh TA, Niendam TA, Minzenberg MJ, Carter CS. Cognitive control deficits in schizophrenia: mechanisms and meaning. Neuropsychopharmacology 2011; 36: $316-38$.

4 Tregellas JR, Smucny J, Eichman L, Rojas DC. The effect of distracting noise on the neuronal mechanisms of attention in schizophrenia. Schizophr Res 2012; 142: 230-6.

5 Verleger R, Talamo S, Simmer J, Smigasiewicz K, Lencer R. Neurophysiological sensitivity to attentional overload in patients with psychotic disorders. Clin Neurophysiol 2013; 124: 881-92.

6 Smucny J, Rojas DC, Eichman LC, Tregellas JR. Neural effects of auditory distraction on visual attention in schizophrenia. PLOS ONE 2013; 8 e60606

7 Weiss EM, Siedentopf C, Golaszewski S, Mottaghy FM, Hofer A, Kremser $C$, et al. Brain activation patterns during a selective attention test-a functional MRI study in healthy volunteers and unmedicated patients during an acute episode of schizophrenia. Psychiatry Res 2007; 154: $31-40$

8 Sugranyes G, Kyriakopoulos M, Dima D, O'Muircheartaigh J, Corrigall R, Pendelbury $G$, et al. Multimodal analyses identify linked functional and white matter abnormalities within the working memory network in schizophrenia. Schizophr Res 2012; 138: 136-42.

9 Ridderinkhof KR, van den Wildenberg WP, Segalowitz SJ, Carter CS. Neurocognitive mechanisms of cognitive control: the role of prefrontal cortex in action selection, response inhibition, performance monitoring and reward-based learning. Brain Cogn 2004; 56: 129-40.

10 Minzenberg MJ, Laird AR, Thelen S, Carter CS, Glahn DC. Meta-analysis of 41 functional neuroimaging studies of executive function in schizophrenia. Arch Gen Psychiatry 2009; 66: 811-22.

11 Ungar L, Nestor PG, Niznikiewicz MA, Wible CG, Kubicki M. Color Stroop and negative priming in schizophrenia: an fMRI study. Psychiatry Res 2010; 181 24-9.

12 Potkin SG, Turner JA, Brown GG, McCarthy G, Greve DN, Glover GH, et al. Working memory and DLPFC inefficiency in schizophrenia: the FBIRN study. Schizophr Bull 2009; 35: 19-31.

13 Weiss EM, Golaszewski S, Mottaghy FM, Hofer A, Hausmann A, Kemmler G et al. Brain activation patterns during a selective attention test-a functional MRI study in healthy volunteers and patients with schizophrenia. Psychiatry Res 2003; 123: 1-15.

14 Edwards BG, Barch DM, Braver TS. Improving prefrontal cortex function in schizophrenia through focused training of cognitive control. Front Hum Neurosci 2010; 4: 32.

15 Vercammen A, Morris R, Green MJ, Lenroot R, Kulkarni J, Carr VJ, et al. Reduced neural activity of the prefrontal cognitive control circuitry during response inhibition to negative words in people with schizophrenia. J Psychiatry Neurosci 2012; 37: 379-88.

16 Brenner CA, Krishnan GP, Vohs JL, Ahn WY, Hetrick WP, Morzorati SL, et al. Steady state responses: electrophysiological assessment of sensory function in schizophrenia. Schizophr Bull 2009; 35: 1065-77.

17 Rissling AJ, Park SH, Young JW, Rissling MB, Sugar CA, Sprock J, et al. Demand and modality of directed attention modulate pre-attentive€ sensory processes in schizophrenia patients and nonpsychiatric controls. Schizophr Res 2013; 146: 326-35.

18 Patterson JV, Hetrick WP, Boutros NN, Jin Y, Sandman C, Stern H, et al. P50 sensory gating ratios in schizophrenics and controls: a review and data analysis. Psychiatry Res 2008; 158: 226-47.
19 Mayer AR, Ruhl D, Merideth F, Ling J, Hanlon FM, Bustillo J, et al. Functional imaging of the hemodynamic sensory gating response in schizophrenia. Hum Brain Mapp 2013; 34: 2302-12.

20 Backes V, Kellermann T, Voss B, Kramer J, Depner C, Schneider F, et al Neural correlates of the attention network test in schizophrenia. Eur Arch Psychiatry Clin Neurosci 2011; 261: S155-60.

21 Baier B, Kleinschmidt A, Muller NG. Cross-modal processing in early visua and auditory cortices depends on expected statistical relationship of multisensory information. J Neurosci 2006; 26: 12260-5.

22 Weissman DH, Warner LM, Woldorff MG. The neural mechanisms for minimizing cross-modal distraction. J Neurosci 2004; 24: 10941-9.

23 Gardner DM, Murphy AL, O'Donnell H, Centorrino F, Baldessarini RJ. International consensus study of antipsychotic dosing. Am J Psychiatry 2010; 167: 686-93.

24 Mayer AR, Wilcox CE, Teshiba TM, Ling JM, Yang Z. Hyperactivation of the cognitive control network in cocaine use disorders during a multisensory Stroop task. Drug Alcohol Depend 2013; 133: 235-41.

25 Mayer AR, Teshiba TM, Franco AR, Ling J, Shane MS, Stephen JM, et al. Modeling conflict and error in the medial frontal cortex. Hum Brain Mapp 2011; 33: 2843-55.

26 Miller GA, Chapman JP. Misunderstanding analysis of covariance. J Abnorm Psychol 2001; 110: 40-8.

27 Power JD, Barnes KA, Snyder AZ, Schlaggar BL, Petersen SE. Spurious but systematic correlations in functional connectivity MRI networks arise from subject motion. Neuroimage 2012; 59: 2142-54.

28 McKiernan KA, Kaufman JN, Kucera-Thompson J, Binder JR. A parametric manipulation of factors affecting task-induced deactivation in functional neuroimaging. J Cogn Neurosci 2003; 15: 394-408.

29 Beauchamp MS, Argall BD, Bodurka J, Duyn JH, Martin A. Unraveling multisensory integration: patchy organization within human STS multisensory cortex. Nat Neurosci 2004; 7: 1190-2.

30 Smucny J, Olincy A, Eichman LC, Lyons E, Tregellas JR. Early sensory processing deficits predict sensitivity to distraction in schizophrenia. Schizophr Res 2013; 147: 196-200.

31 Tregellas JR, Ellis J, Shatti S, Du YP, Rojas DC. Increased hippocampal, thalamic, and prefrontal hemodynamic response to an urban noise stimulus in schizophrenia. Am J Psychiatry 2009; 166: 354-60.

32 Arce E, Leland DS, Miller DA, Simmons AN, Winternheimer KC, Paulus MP. Individuals with schizophrenia present hypo- and hyperactivation during implicit cueing in an inhibitory task. Neuroimage 2006: 32: 704-13.

33 Ludewig K, Geyer MA, Vollenweider FX. Deficits in prepulse inhibition and habituation in never-medicated, first-episode schizophrenia. Biol Psychiatry 2003; 54: 121-8

34 Lewis-Hanna LL, Hunter MD, Farrow TF, Wilkinson ID, Woodruff PW. Enhanced cortical effects of auditory stimulation and auditory attention in healthy individuals prone to auditory hallucinations during partial wakefulness. Neuroimage 2011; 57: 1154-61.

35 Kompus K, Falkenberg LE, Bless JJ, Johnsen E, Kroken RA, Krakvik B, et al. The role of the primary auditory cortex in the neural mechanism of auditory verbal hallucinations. Front Hum Neurosci 2013; 7: 144

36 Regenbogen C, De VM, Debener S, Turetsky BI, Mossnang C, Finkelmeyer A, et al. Auditory processing under cross-modal visual load investigated with simultaneous EEG-fMRI. PLOS One 2012; 7: e52267.

37 Vita A, De PL, Deste G, Sacchetti E. Progressive loss of cortical gray matter in schizophrenia: a meta-analysis and meta-regression of longitudinal MR studies. Transl Psychiatry 2012; 2: e190.

38 Clasen PC, Beevers CG, Mumford JA, Schnyer DM. Cognitive control network connectivity in adolescent women with and without a parental history of depression. Dev Cogn Neurosci 2014; 7: 13-22. 